\title{
Exploring the Activity and Stability of Pt-based Catalysts through Analytical Electron Microscopy
}

David A. Cullen ${ }^{1}$, Deborah Myers ${ }^{2}$, Jeffrey Greeley ${ }^{3}$, Jonah Erlebacher ${ }^{4}$, Grant Thoma ${ }^{5}$ and Andrew J. Steinbach ${ }^{5}$

1. Materials Science \& Technology Division, Oak Ridge National Laboratory, Oak Ridge, TN USA

2. Chemical Sciences and Engineering Division, Argonne National Laboratory, Argonne, IL USA

3. Davidson School of Chemical Engineering, Purdue University, Lafayette, IN USA

4. Whiting School of Engineering, Johns Hopkins University, MD USA

${ }^{5 .}$ Fuel Cell Components Program, 3M Company, MN USA

Electrocatalysis for energy storage and conversion often relies on rare and costly precious metal catalysts. This is particularly true for low-temperature fuel cells, where Pt serves as both the anode and cathode catalyst for the hydrogen oxidation reaction and oxygen reduction reaction, respectively.[1] Decreasing the overall catalyst loadings while maintaining both performance and stability is a critical and intense area of research. Electron microscopy techniques, ranging from high-angle annular darkfield (HAADF) imaging to 3D tomography, have been instrumental in the development of catalysts capable of meeting these aggressive targets.[2,3]

This work presents a unique strategy for catalyst optimization by combining a Pt-transition metal alloy with a corrosion-resistant whisker support. These materials, dubbed ultra thin films (UTF), mark the state-of-the-art in electrocatalysis for the oxygen reduction reaction in polymer electrolyte membrane fuel cells. As shown in Figure 1, the UTF catalyst consists of a high aspect ratio perylene-red support coated with a thin, polycrystalline layer of Pt or Pt-alloy. This geometry adopts the stability and high specific area of model bulk films, leading to a highly active catalyst which is considerably more stable than the nanoparticle counterpart.

New challenges have arisen as the total Pt loading has been pushed to ultra-low limits. As shown in Figure 2, as the precious metal loading drops below $20 \mu \mathrm{g} / \mathrm{cm}^{2}$, the thin films become discontinuous and the structure collapses during fuel cell tests. To address this problem, various structural and chemical modifications are being explored. This work will present how electron microscopy and synchrotronrelated techniques have been combined with modeling and electrochemical testing to develop electrocatalysts which maintain high fuel cell performance at a fraction of the metal loading. [4]

References:

[1] A.J. Steinbach et al, ECS Transactions, 69 (2015) 291-301.

[2] D.A. Cullen et al, Journal of Materials Chemistry A, 3 (2015) 11660-11667.

[3] B.T. Sneed et al, ACS Applied Materials \& Interfaces, 9 (2017) 29839-29848.

[4] Research sponsored by the Fuel Cell Technologies Office, Office of Energy Efficiency and Renewable Energy, U.S. Department of Energy (DOE) and ORNL's Center for Nanophase Materials Sciences (CNMS), which is a DOE Office of Science User Facility 

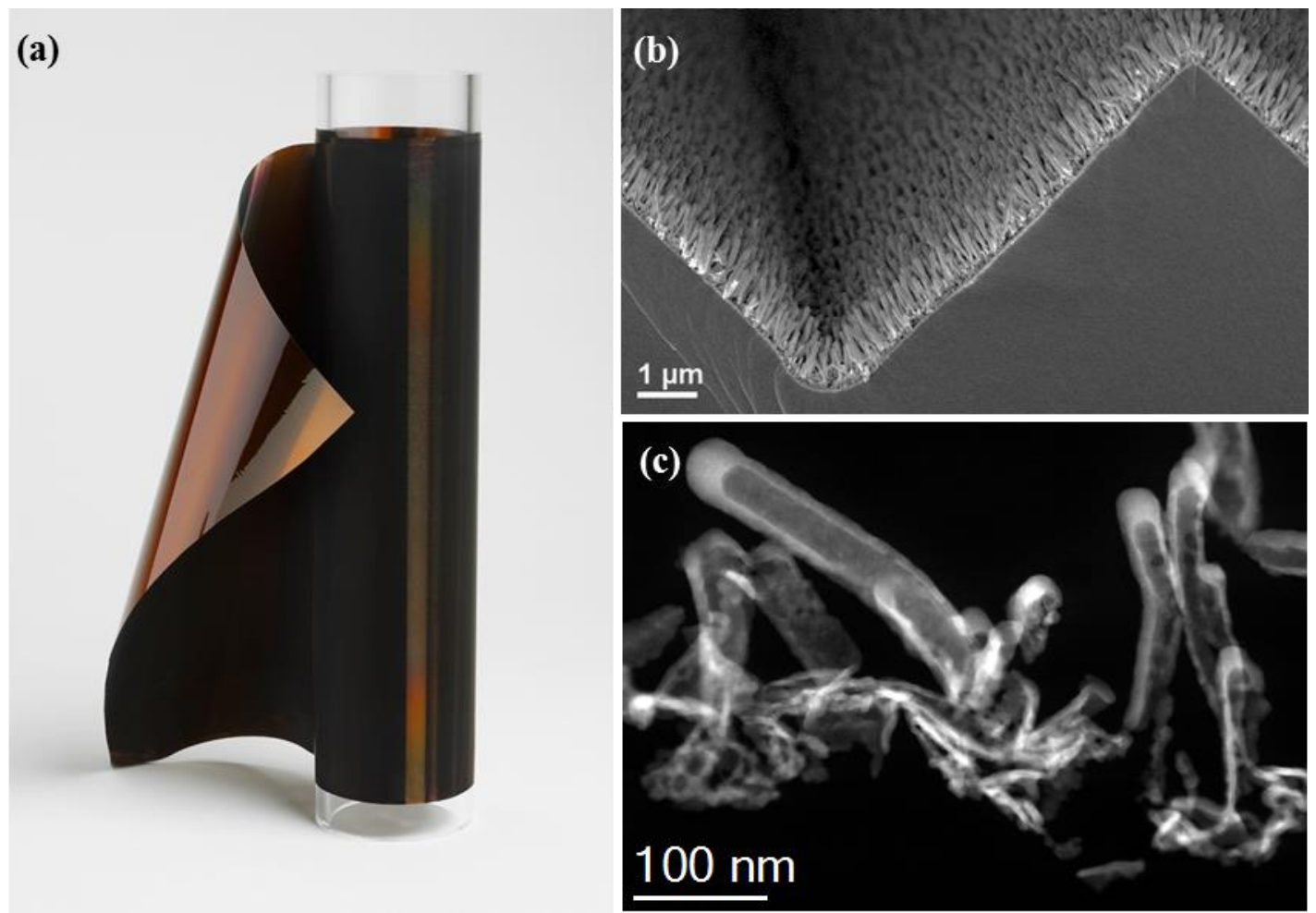

Figure 1. (a) Photograph, (b) secondary-electron image and (c) HAADF-STEM image of UTF electrocatalysts.
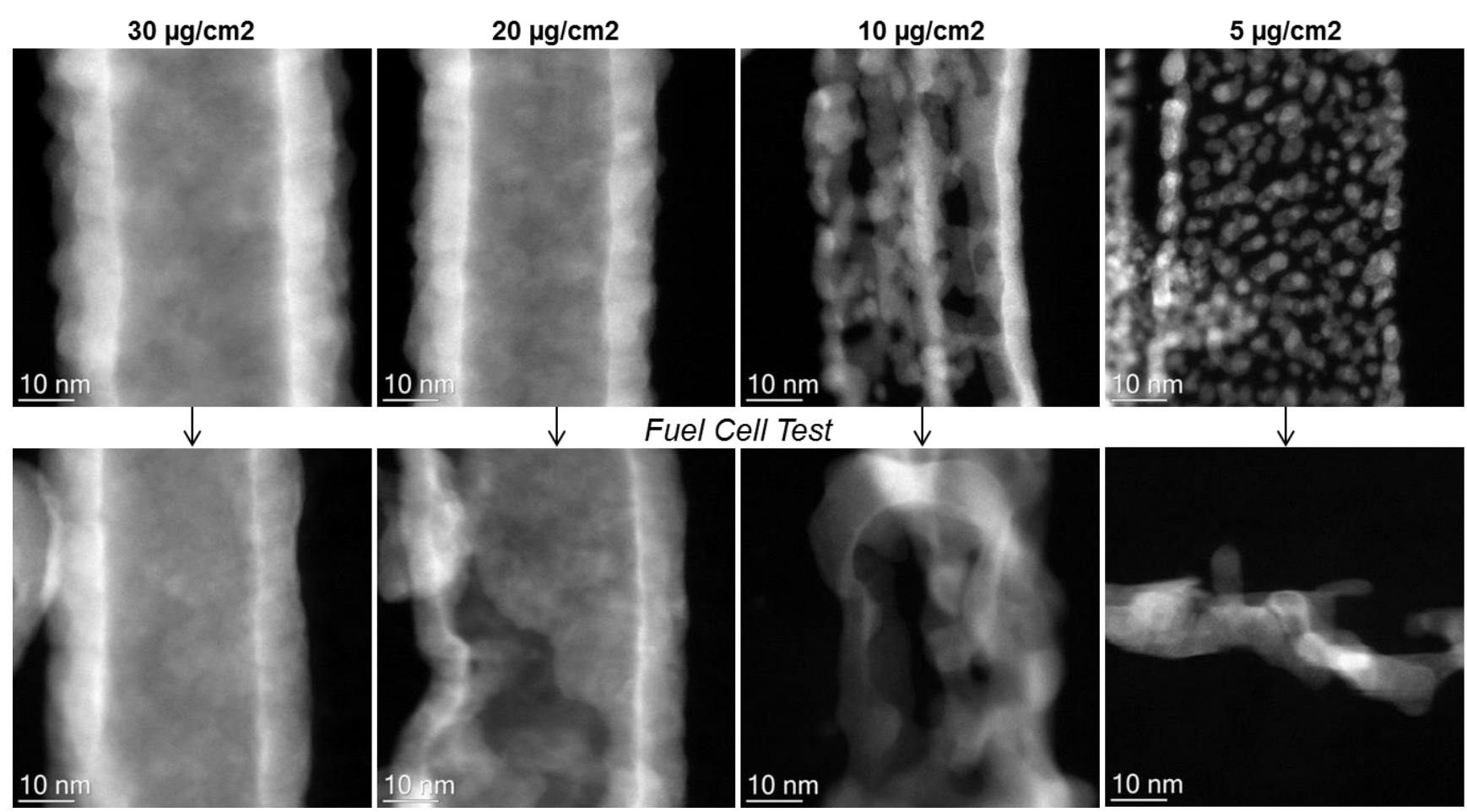

Figure 2. HAADF-STEM images of Pt-alloy UTF at four different Pt loadings both (top) before and (bottom) after fuel cell testing. At ultra-low loadings, the alloy does not fully wet the whisker support, leading to the collapse in the structure and loss in performance during fuel cell tests. 